\title{
Epidermodysplasia Verruciformis: Functional Role of E6 and E7 Oncoproteins as the Link between Viral Infection to Carcinogenesis
}

\author{
Aadrita Hazra, Pritha Bose, Shakti Prasad Pattanayak* \\ Division of Pharmacology, Department of Pharmaceutical Sciences and Technology, Birla Institute of Technology, Mesra, Ranchi, \\ Jharkhand, INDIA.
}

\begin{abstract}
Background: Cancer is the most dreadful disease since last few decades. Besides all causative reasons, it is observed that one in every five cancers globally is linked with infectious diseases. Among all the infectious oncogenic viruses, 35\% of the human cancers have been caused by Human Papilloma Viruses (HPVs). One such type is Epidermodysplasia Verruciformis (EV), a tropical disease also known as the Tree man syndrome. Hypothesis: $E V$ is one of the most potential illustrations linking viral infection with skin carcinogenesis. The HPV virus acts as a cofactor with the UV radiation. The lesions caused by invasion of the HPV in the sun exposed wounded areas of the EV patients clearly links $\beta$ HPV and cellular proliferation where EVER1 and EVER2 gene mutations are equally cardinal. When the viral infection persists, the somatic mutations over time accumulate, leading to precancerous lesions at first, followed by the malignant transformation. Two out of the eight proteins that the HPV genome codes for namely E6 and E7, account for the high-risk type HPVs that cause cancer. Both work similarly but on different sites. Both E6 and E7 proteins encode the main HPV's oncoprotein which promotes cell progression and viral replication. Results and Conclusion: The role of E6 and/or E7 in tumor progression, metastasis promotion, NOTCH and TGF $\beta$ pathway modification, DNA methyltransferase stimulation, histone modification, E-cathedrine modification etc. have been described in this review for the better understanding of the linkage between viral infection and carcinogenesis process.
\end{abstract}

Key words: Epidermodysplasia Verruciformis, HPV, Skin Carcinogenesis, EVER genes, E6 and E7 oncoproteins.

\section{INTRODUCTION}

Cancer is one of the most widespread and deleterious disease that has now become way beyond the facile concept of uncontrolled cell growth. Among the causative factors such as, UV radiations, tobacco, $\gamma$-rays or mutations, one out of every five cancers have been found to be linked with infectious diseases. The oncogenic viruses that have been identified till date are: Hepatitis B, herpes virus, Epstein-Barr virus, Human papilloma virus, Merkel cell polyomavirus and Kaposi's sarcoma virus and human T-cell lymphoma virus and about $35 \%$ of the human cancers have been caused by HPV. ${ }^{1}$
The papillomaviruses infect the cutaneous and the mucosal epithelium thereby causing proliferative lesions. ${ }^{2}$ The epidermological findings indicate that HPV may be necessary in the initial stage and may become dispensable once the non-melanoma skin cancer (NMSC) has been fully established. ${ }^{3}$ The NMSC encompasses the basal cell carcinoma (BCC) and squamous cell carcinoma (SCC) that occur most frequently globally. One such type is Epidermodysplasia Verruciformis (EV), also known as the Tree Man Syndrome.
Submission Date: 06-11-2019; Revision Date: 11-01-2020; Accepted Date: 14-02-2020

DOI: 10.5530/ijper.54.2.29 Correspondence: Dr. Shakti Prasad Pattanayak

Cancer Research Group, Division of Pharmacology, Department of Pharmaceutical Sciences and Technology, Birla Institute of Technology, Mesra, Ranchi-835215, Jharkhand, INDIA. Phone: +91-6512275444 E-mail: sppattanayak@ bitmesra.ac.in

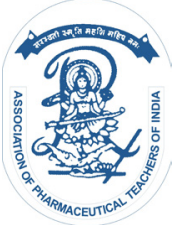

www.ijper.org 


\section{Epidemiology}

Most of the cancers, diagnosed at a young age, are mostly associated with higher HPV prevalence across ethnic groups. The presence of HPV has been detected in oral cavity cancer $(32.0 \%)$, anal cancer $(91.1 \%)$, cervical cancer $(90.6 \%)$, oropharyngeal cancer $(75.0 \%)$, vulvar cancer (68.8\%), laryngeal cancer $(20.9 \%)$ and penile cancer $(63.3 \%){ }^{4}$ However, EV has been considered to be one of the most potential illustrations that links skin carcinogenesis to the viral infection caused by HPV. ${ }^{1}$ Epidermodysplasia Verruciformis is a very rare genodermatosis and was first documented in 1922 by Lewandowsky and Lutz. ${ }^{5}$ So far, $501 \mathrm{EV}$ cases have been globally reported. ${ }^{6} \mathrm{EV}$ cases have been found to be either sporadic or inherited (autosomal recessive, sex linked or autosomal dominant). In fact, in one third of the patients, cancer has been seen to develop in the light exposed areas. ${ }^{7} \mathrm{EV}$ cases have been seen from Europe, Poland, Latin America. EV usually begins in childhood or even infancy. ${ }^{8}$ This cancer has its average onset at the age of 31 years but it can even be as early as 13 years, ${ }^{7}$ where the malignancy becomes definitive in the fourth or fifth decade. ${ }^{8}$

\section{Phenotype of EV}

$\mathrm{HPV}$ infections in EV patients are asymptomatic and are manifested as polymorphic skin lesions that look like tree branches emerging from human body. Its phenotype relies on the immunological and viral features that characterize the EV patients. The infections lead to the development of pathognomonic pityriasis versicolor-like lesions; flat warts like lesions. Such lesions are generally reddish brown in colour. Papular lesions are usually found in the trunk, face and neck region and resemble verrucae planar. Pityriasis-like warts are found to dominate over the flat warts. The benign lesions are usually found on the torso region that appear as scaly and irregular. On the other hand, the malignant ones are found usually in the exposed areas of the skin. ${ }^{9}$ These warts usually appear as disseminated polymorphic cutaneous lesions. ${ }^{1}$ Over the time, such manifestations have been seen to be progressing to NMSC or SCC especially in the sun exposed areas. ${ }^{10}$ Histological studies of such lesions have revealed kolocytes, parakeratosis, acanthosis, hyperkeratosis and keratinocytes having pale stained cytoplasm. ${ }^{11} \mathrm{EV}$ carcinoma cases were reported to retain a high number of reprints of HPV genomes which were found to be maintained as episomes expressing the oncogenic transcripts E7 and E6. ${ }^{1}$

\section{Pathogenesis of EV HPV}

The human papillomaviruses are non-enveloped viruses belonging to the family papilomaviridae. ${ }^{12}$ They have a double stranded circular DNA (8000 nucleotide base pairs with nine open reading frames. The icosahedral capsid that consists of 72 capsomeres encloses the viral DNA, associated with the histones that form minichromosomes. The classification of HPVs is done on the basis of the ORFs nucleotide sequence of the L1 capsid. The HPVs have been divided into 16 genera for instance-mu, nu, alpha, beta, gamma where most of them belong to the alpha and beta genuses. ${ }^{13}$ The HPV genome has two regions-the coding region that encodes for early (E) and late (L) proteins and the non-coding region (NCR) or the long coding region (LCR). When the initial phases of viral replication occur, the early proteins, E1, E2, E4, E5, E6 and E7 are expressed to regulate the plasmid replication and its expression along with the cell adaptation. The late protein on the other hand forms the structural unit of capsid and encodes L1 (major) and L2 (minor) capsid proteins. ${ }^{14-16}$ The LCR portion of the genome comprises of $10 \%$ to $15 \%$ of the whole genome and it is placed towards the E region as shown in the Figure 1. It has binding sites for several transcription factors that are important for the viral protein expression. ${ }^{17}$

\section{HPVs as epidermis invader}

HPV has been found to inhabit the cutaneous epithelium, targeting the hair follicles. HPV5 and HPV8 both belonging to the $\beta$-genus of the family have been found frequently in the lesions of the EV patients leading to skin carcinoma. ${ }^{18}$ The $\beta$ HPV's have been found to infect he stratified epithelium and link their life cycle with keratinocytes' differentiation. They begin by approaching the basal cells of the stratified epithelium that proliferates because of any micro-abrasion or micro-injury. The process of viral entry proceeds by the interaction with the cell surface heparin sulphate which is followed by endocytosis-mediated by clathrin or caveola. ${ }^{19}$ When the virus exits the basal layer, epithelial cells stop proliferating, therefore initiating differentiation programme. However, HPV requires delayed differentiation to ensure its replication in the upper granular and the suprabasal layer. ${ }^{1}$ A potential role of the DNA repair process has been seen to integrate with the HPV infection, ${ }^{17}$ where HPV acts as a cofactor along with the UV radiation that helps to link the $\beta$-HPV and cellular proliferation where EVER1 and EVER2 mutations are equally cardinal. ${ }^{20}$ 


\section{The EVER genes}

It has been found that specific events occurring in somatic cells of the hosts are also important for the malignant transformation. Viral infection alone can't be the only cause for malignancy. When the viral infection persists, the somatic mutations over the time, accumulate, leading to precancerous lesions at first, followed by the malignant transformation.

Transmembrane channel (TMCs) like proteins have been found to concatenate HPV, carcinoma and EV, playing an important role in controlling the link between viral homeostasis and the damage caused by the UV. Two loci-EV1 and EV2 were found to be susceptible to EV that helped to ascertain the two novel genes EVER1 and EVER2. ${ }^{21,22}$ These two genes have been found to code for a transmembrane protein in the endoplasmic reticulum that functions as an ion transporter. ${ }^{23}$ The EVER1 gene was found to be identical with the TMC6 and the EVER2 gene, identical to the TMC8. ${ }^{24,25}$

The EVER1 gene was found to be responsible for maintaining zinc homeostasis. EVER1 protein has ten domains, two leucine-zipper motifs and two putative glycosylation sites. It consists of 20 exons, 19 introns and encodes for 4 transcripts. EVER 2 protein was found to have eight domains, three leucine-zipper motifs and two putative glycosylation sites. It consists of 16 exons, 15 introns and encodes for just one transcript with 4419 base-pairs. Something to be accentuated here is that the locus for EVER gene was found in a region with locus PSORS2 that was susceptible to psoriasis. ${ }^{5}$ Several cases of mutations have been reported in EVER genes like splice site mutation, nonsense mutation, single nucleotide polymorphism and frameshift mutation that have contributed to the pathogenesis of $\mathrm{EV}$.

\section{Physiology of EVER genes}

The element zinc plays a vital role in acting as a secondary messenger in signal transduction. Hence, is homeostasis is required. Zinc transporters are the transmembrane proteins that maintain the balance of zinc efflux and influx, regulating the action of zinc as secondary messenger. Zinc transporters fall under two categories-SLC39, called as Zrt i.e. ZIP and SLC30 designated as cation diffusion facilitator $(\mathrm{CDF})$ or as the zinc transporter $(\mathrm{Zn}-\mathrm{T}) .{ }^{1,26}$

ZIP and Zn-T counter-balance each other's work and protect the cell from zinc toxicity. The former is responsible for translocating zinc towards the cytoplasm whereas the latter translocates zinc from the nucleus, outside the cytoplasm. EVER genes have a primary role in maintaining this zinc homeostasis. The EVER genes form a complex with the $\mathrm{Zn}-\mathrm{T}$ and translocate the excess zinc ions from the pool of free zinc ions to the lumen of endoplasmic reticulum thereby, decreasing the zinc concentration in the nucleus. So in a way, the EVER/Zn-T complex regulates the activity of many transcription factors by modulating the zinc efflux and influx. ${ }^{27}$ When the cell surface gets triggered, free zinc ions get released from the endoplasmic reticulum, causing a phenomenon called as the zinc wave followed by the binding of the zinc ions to the zinc finger. Zinc fingers are the cysteine-based protein domains to which the zinc ions bind to. Zinc fingers are specifically the Cys2His 2 motifs (CCHH). ${ }^{1}$ Zinc ions bind to the thiolate group of the cysteine residues of the enzyme phosphatase, suppressing its activity and phosphorylating a number of transcription factors thus, initiating signal transduction. The EVER/Zn-T complex is also able to suppress the AP-1, Fos, Elk-1 transcription factors as shown in Figure 3. This affects the lymphocytes' maturation, differentiation along with cytokine expression that have been infected by viral proteins. Hence in any way, the EVER proteins' inactivation can either compensate for the absence of viral function or it is the virus that tends to obstruct the functioning of EVER genes causing dysregulation in the signal transductions. The EVER/Zn-T complex influences the transactive domain of c-Jun as well. Jun is a constituent ofAP-1 dimer that if getting phosphorylated by GSK3 gets suppressed and gets activated if gets phosphorylated by JNK. The G3K3 (glycogen synthase kinase-3) is involved in a lot of pathways such as NOTCH, Wnt/ $\beta$-catenin and Hedgehog. ${ }^{28}$ The mutated EVER/Zn-T complex modulates the JNK activity in a GSK-3 independent way thereby inhibiting the transactive domain of c-Jun. This activation of the cell proliferation pathway favours even the replication of keratinocytes that get infected by the HPVs. ${ }^{5}$ Free zinc is also able to induce the stimulation of c-Jun, $\mathrm{N}$ terminal kinase signalling pathway via the PI3K pathway. Hence, JNK stimulation is also stimulated by PI3K-Rac-1. In a nutshell, zinc exposure leads to the phosphorylation of JNK, p38 MAPK and ERK and this stimulation leads to the overproduction of proinflammatory cytokines which are conducive for the virus infected cells and their sustenance. Therefore, the EVER-Zn-T complex is responsible for the regulation of zinc induced transcription factors.

The EVER2 has also been found to be linked with abnormality in the protein profile of TRAF2. EVER gen interacts with TRADD impeding the recruitment of RIPK1 and TRAF preventing TRAF2's ubiquitination so that it can associate with Fas-associated protein with death domain (FADD), activating the caspase promoting TRAIL induced apoptosis through RIPK1's 
death domain. Hence, EVER2 prevents the impairment of NF-KB pathway. ${ }^{5}$ However, in case of the mutated EVER2 gene, the TRAF2 is able to bind to the TRAF2 binding site of TRADD in case of TNF- $\alpha$ stimulation and forming a TRADD/RIPK1/TRAF2/CIAPs complex I that resists the TRAIL induced apoptosis and induces NF-KB cascade. ${ }^{29}$

CIB1 is calcium-integrin binding protein 1 which is an intracellular binding partner of a platelet-specific $\alpha$ integrin cytoplasmic tail. It is involved in Calcium signalling, migration, proliferation and cell survival. Binding partners of CIB1 are- p-21 activated kinase (PAK1), serine threonine kinase. Apoptosis signal regulating kinase1 (ASK1) and polo-like-kinase (PLK3). CIB1 has also been found to support the PI3K/AKT and MEK/ ERK oncogene pathway. ${ }^{30}$ Due to the variations in the EVER1 and the EVER2 genes, low levels of CIB1 was observed. Through co-immunoprecipitation studies, it was found that EVER1, EVER2 and CIB1 formed a multimeric complex. CIB1 was found to interact with the gamma-HPV4 E8 and alpha HPV16 E5. The complex CIB1/EVER1/EVER2 restricts the HPV expression. ${ }^{31}$ However, the CIB1 does not affect the zinc transport or the NF-kB. No interaction has been seen in the CIB1 and the $\mathrm{Zn}-\mathrm{T}$ protein. ${ }^{32}$

\section{The viral oncoproteins}

E6 and E7 are the two oncoproteins coded by the HPV genome that promotes cell cycle progression and viral replication. They both work similarly but on different sites. They contribute to the carcinogenesis by different processes, making the host genome unstable, causing chromosomal aberrations, thereby interfering with mitosis and inhibiting the tumor suppressor gene. In fact, E6 and E7 induce the accumulation of damaged DNA. E6 and E7 bring out such events through the presence of class 1 PDZ (PSD-95/Dig/ZO-1) binding motif which is present at the carboxy terminal of the viral oncogene that inducts malignancy. PDZ binding activity is regulated by the phosphorylation with the help of protein kinase A (PKA)/AKT that inhibits the recognition of PDZ. ${ }^{33}$

E6 is primarily responsible for impairing p53, favouring the anti-apoptotic pathways whereas E7 is responsible for reprogramming the epithelial cells at the surface of epithelium, letting the host cell re-enter the cell cycle. This provides a conducive milieu for the viral replication that ultimately destabilizes the host genome. The focal amplification of the loci having the following genes have been found in the unstable host genome- 7p11.2-epidermal growth factor receptor (EGFR), 3q24.1- tumor growth factor $\beta$ receptor 2 (TGF $\beta$ R2), 10q23.31-phos- phatase and tensin homolog (PTEN), 3q28-tumor protein (TP63), 18q1.2-SMAD family member 4(SMAD 4). The presence of HPV in the SCCs has shown losses at $11 \mathrm{q}, 3 \mathrm{p}$ and gains at $3 \mathrm{q}$ locus. In $50 \%$ of the cancers, amplification of high frequency has been seen at $3 q$ that contains the catalytic $\alpha$ subunit of the phosphatidyl inositol-4,5-bisphosphate-3-kinase (PIK3CA), the most common mutated gene in HPV cancers. ${ }^{17}$

\section{Role of Type 1 Interferons (IFNs)}

Type 1 interferons belong to the family of cytokines and are involved in the inhibition of cell division and they interfere with the viral replication as well. Type1 interferon was found to suppress the viral E6 and E7 transcription. IFN $-\alpha$ and $\beta$, the most dominating interferons which are activated because of the cellular transcription factors-IRF-3 and IRF-7, the interferon regulatory factors. Both acts differentially on the Type1 IFN genes. IFR-7 induces activation of IFN $\alpha$ and IFN $\beta$ whereas IRF-3 is the potential activator of IFN $\beta$ only. IRF-7 was found to stimulate the HPV transcription whereas IRF-3 and its activators (Poly (I:C) and 5'ppRNA) suppressed the expression of cutaneous virus. E6 oncoprotein was found to bind to the IRF-3 that decreased its tumor suppressor activity. E7 oncoprotein was found to bind with IRF-1. The suppression of the tumor suppressor activity favoured the IRF-7 to dominate as a promoter of tumor promoter and therefore it promotes cutaneous viral expression. ${ }^{34}$

\section{Role of E6 in tumor progression}

P53 is a signalling protein that is responsible for regulating the large subsets of the target proteins. It can sense the genotoxic stress and stress induced by the DNA damage. ${ }^{20}$ It controls the negative regulators of the cell cycle progression like p-21, 14-3-3, GADD45 $\alpha$ and the pro-apoptotic proteins like PUMA, BAK and BAX. MDM2 acts as its antagonist by working as a ubiquitin ligase. Studies show that E6 targets the $\mathrm{p} 53$ by a variety of mechanisms (Figure 7). E3 ubiquitin ligase (E6AP/ UBE3A), forms complex with E6 leading to the proteosomal degradation of $\mathrm{p} 53 . \Delta \mathrm{NP} 73$ is a $\mathrm{p} 53$ isoform that antagonizes p53. Some species like $\beta H P V 38$, have been found to accumulate $\triangle \mathrm{NP} 73$ that by antagonizing p53 affects apoptosis by impairing the apoptotic proteins like BAX, FAS, P21, CHKs etc. ${ }^{35}$ The other $\beta \mathrm{HPV}$ species for example: HPV23 impedes the phosphorylation dependent activation of P53. E6 also was found to inhibit HIPK2. HIPK2's activity is goaded by UV. It is a protein kinase that is responsible for the phosphorylation at p53 at Ser 46. It therefore acetylates it at Lys382 and promotes its expression. E6 is also found 
to promote the activity of human telomerase reverse transcriptase (hTERT). ${ }^{36}$ E6 can interact with $\mathrm{Bcl}-2$ Homologous antagonist killer (BAK), the pro-apoptotic protein. It prevents $\mathrm{BAK}$ to accumulate in response to UV and hence prevent apoptosis. E6 attenuates the transition from the G1 to the S-phase by the check points, therefore favouring the cellular proliferation with the damaged DNA. This is due to E6's interaction with the acetyl transferase p300 causing decrease in p300..$^{35}$ The E6 oncoprotein of the $\beta H P V$ was found to suppress the two tumor suppressor (NOTCH and TGF $\beta$ ) pathways. E6 tends to bind to the MAML1 and SMAD2 and 3 proteins that act as cofactors for the transcription process of NOTCH and TGF- $\beta$ respectively. ${ }^{37}$

\section{Role of E6 in promoting metastasis by suppressing P63 and micro-RNA}

MicroRNA is a key regulator of epidermal proliferation. However, it gets downregulated in HPV infection. ${ }^{38}$ MicroRNAs are the small molecules of RNA that can cause an impact on the protein expression at the translational level. It is involved in the regulation of cellular pathways. Its dysregulation can exert carcinogenic effects. These small RNAs suppress the unwanted genetic materials for the normal operation of the cell. The loss of microRNAs can prevent the suppression of tumorigenesis. MicroRNAs are primarily responsible for controlling the apoptotic pathway and the cell cycle regulation. ${ }^{39}$ When the basal cells transform into the suprabasal cells, they tend to lose their dividing potential and hence undergo the terminal differentiation process. This process is managed by the microRNA-203. It induces the cell cycle exit and therefore promote differentiation in the epithelial cells. MiR-203 and p63 act as regulators of the epidermal homeostasis that gets disrupted during the HPV invasion. ${ }^{38}$ p 63 is a protein homolog of the p53 tumor suppressor gene, that has two promoter regions encoding two isoforms-TP63, that contains the $\mathrm{N}$-terminal trans activity domain and the other, lacking TA $\triangle \mathrm{Np} 63 .{ }^{40}$ Among the two isoforms, $\Delta \mathrm{Np} 63$ is the more dominant one in the basal keratinocytes. $\Delta \mathrm{Np} 63$ has a sterile $\alpha$ motif domain (SAM), a terminal inhibitory domain, that reduces the activity of p63. ${ }^{41}$ It hence plays an important role in maintaining the stemness and the proliferating ability of the cell that suppressed the differentiation factor involucrin. It tends to inhibit the TA's activity by either heterodimerization or by competing for the consensus promoter. ${ }^{40} \mathrm{Mdm} 2$ binds to $\triangle \mathrm{NP} 63$ and promotes its translocation to the cytoplasm and degrading it. ${ }^{41}$ Studies have shown that $\mathrm{p} 63$ functions as tumor suppressor especially the TAP63 isoform. The TAP63 activates p53 leading to the arrest of cell cycle and hence apoptosis. CCAAT/enhancer binding protein $\alpha(\mathrm{C} / \mathrm{EBP} \alpha)$, a transcription factor that is involved in the regulation of differentiation process. It suppresses the UV induced skin carcinogenesis. It has its hairpin region to which miR-203 gene directly binds to the $\Delta \mathrm{Np} 63 \alpha$. The miR203 targets the $\Delta \mathrm{Np} 63$. The $\mathrm{C} / \mathrm{EBP}$ is itself regulated by p300 which is its co-activator. It is a histone acetyl transferase and histone acetylation process is linked to the upregulation of $\mathrm{C} / \mathrm{EBP} \alpha \cdot \mathrm{p} 300$ is knocked down by the $\mathrm{E} 6$ oncogene that decreases the expression of the $\mathrm{C} / \mathrm{EBP} \alpha$ transcription factor $\mathrm{p} 300.38,42$ This causes their degradation which inhibit the ATM and the ATR, driving carcinogenesis. ${ }^{43} \mathrm{E} 6$ was found to counteract the downregulation of tumor promoting $\Delta \mathrm{Np} 63 \alpha$ and the upregulation of tumor suppressor and miR-203. PMA can induce the $\mathrm{C} / \mathrm{EBP} \alpha$ transcription factor which is very potent in inducing the differentiation of the keratinocytes which is also an important function in response to the UV induced carcinogenesis. The miR regulates IL-8 while E6 suppresses the IL-8. HPV E6 upregulates the stemness factor $\Delta \mathrm{Np} 63$ by suppressing miR-203 and increase the cells in the S-phase. The interaction of E6 with p300 has been found to be important for the downregulation of p53 that therefore causes tumorigenesis.

\section{Role of E6 in histone modification}

The E6 oncogene controls the regulation of the two coactivators, histone arginine methyl transferases- PRMT1 and CARM1 that causes the arginine residues to undergo histone methylation. E6 can downregulate the expression of these two methyltransferases in turn affecting the p53 stabilization. E6 mediates the histone methylation by binding to the PRMT1 and CARM1 at the p53 promoter and suppressing the P53 binding to the DNA. E6 can also inhibit the SET7 that catalyses H3K4 mono methylation and methylation of the non-histone proteins including p53 and downregulating p53K372 mono methylation. This decreases the stability of p53. Hence, E6 can modulate SET7, CARM1 and PRMT1 which in turn alters the function of $\mathrm{p} 53 .{ }^{19}$

\section{Role of E6 in Notch pathway}

Notch pathway is a signalling system which is categorized as a contact dependent pathway. ${ }^{44}$ It gets activated by a cell to cell contact process where the ligands that are anchored to the membrane and belong to the Jagged or the Delta family, bind to the Notch receptors. ${ }^{43}$ It is involved in the embryogenesis and in the developmental process of organism. The Notch extracellular truncation fragment is recognized by the gamma 
secretase complex which lead to the proteolytic process. This proteolysis is important for releasing the NICD (Notch intracellular domain) and $\mathrm{N} \beta$ peptide that lets the NICD within the nucleus. Within the nucleus, the NICD interconnects with the DNA-binding transcriptional repressor recombination signal binding protein for the immunoglobulin Kappa $j$ region (RBP-J) by its BBP-J associated molecule (RAM) domain. This interaction leads to the recruitment of mastermind like proteins (MAMLs) to the promoter of the Notch target genes. The ICN/RBPJ/MAML complex represents the core NOTCH transcriptional activator complex and then the MAML conscripts' additional co-activators. ${ }^{43}$ The NOTCH pathway is observed to be an important factor for the keratinocytes' differentiation and its defect has been involved in the cutaneous SCCs. Studies have shown that the E6 gene is able to interact with MAML1 and intracellular cleaved NOTCH1 (ICN1), inhibiting the NOTCH. ${ }^{37} \mathrm{E} 6$ attaches to the LXXLL motif within the $\mathrm{C}$ terminal transactive domain (TAD) 2 of the MAML1 by causing mutations in the MAML protein. ${ }^{43}$ The chromatin immunoprecipitation experiments have shown that E6 can bind to the DNA bound RBPJ/ICN/MAML1 activator complex and inhibit it as shown in Figure 4. Other than MAML1, E6 has also been found to target UBE3A2. The inhibition of NOTCH causes a displacement of EP300/CREBBP from the MAML1 TAD1 that causes dysregulation of the $\mathrm{p} 53 .{ }^{37}$

\section{Role of E6 in TGF- $\beta$ pathway}

The E6 dysregulates the TGF- $\beta$ 's tumor suppressor property by preventing the Smad 4 complex to form complex with the other Smad complexes. There occurs the transcriptional activation of CDK2 inhibitors p21CIPI (CDKN1A) and p27KIPI (CDKN1B) and the CDK4/CDK6 inhibitor p15INK4B (CDKN2B), that are linked to the $\mathrm{G} 1$ phase cell cycle arrest. ${ }^{43}$ The $(T G F-\beta)$ is involved in differentiation, immunological responses and healing of the injured tissues. Any abnormality in this signalling pathway can lead to the inflammatory conditions, fibrotic conditions and even malignancies. This pathway in cancer acts as a tumor suppressor that induces apoptosis inhibiting the cell proliferation. The TGF- $\beta$ family members signal by the type I and type II, dual specificity kinase receptors. When the ligand binding occurs, it induces the oligomerization and phosphorylation of both the receptors. After the activation of the R-SMAD, there is a formation of trimeric complexes where SMAD4 acts as a common mediator which then gets translocated to the nucleus where they work together with the other co- repressors, co-activators and the transcription factors. E6 oncoprotein of the HPV, targets SMAD3 for proteosomal degradation. ${ }^{45} \mathrm{E} 6$ prevents the association of SMAD4 with the SMAD2/Smad3 complex hence preventing its binding to the DNA as shown in Figure 5. SMAD3 and SMAD2, that are the transcriptional cofactors in the pathway that are obstructed by the E6 protein to form a complex with the SMAD4 ${ }^{37} \mathrm{Smad} 2$ and Smad3, they both don't have a definitive LXXLL motif and so, are expected to bind the different E6 sequences. The CDKN28 promoter has a well-defined Smad binding element (SBE). By chromatin immunoprecipitation, it was reported that E6 resulted in the decreased SMAD2/ SMAD3 and Smad 4 complex occupancy at the CDKN28 promoter. Hence, SMAD4's association is disrupted by the impairment of its association with the other cofactors of transcription that form the complex for the initiation of the expression of the TGF- $\beta$ pathway. ${ }^{37}$

\section{DNA damaging response and E6}

Whenever there is any sort of alteration in the DNA by any intrinsic or any extrinsic factor, the DNA damage response gets activated to maintain the genomic integrity. And if the damage cannot be repaired, checkpoints get activated triggering the cell death. DNA damage can be caused due to endogenous factors such as-errors in the replication process, unrepaired single strand lesions and base loss or termination, it is also caused by environmental factors such as-exposure to UV, IR, tobacco and dietary nitrosoamines. DNA repair involves a lot of pathway s to get activated such as base excision repair (BER), non-homologous end joining (NHEJ), mismatch repair etc. UV induced DNA damage involves the formation of intrastrand cross links-usually cyclobutane pyrimidine dimers (CPDs) that can lead to point mutations. Apart from that, crosslink too leads to replication fork collapse which if doesn't get repaired, leads to double strand break (DSB) contributing to chromosomal aberrations. During the S-phase of the cell cycle, to bypass the damaged bases of crosslinked DNA, translesion synthesis occurs that otherwise would have led to the replication fork collapse. Nucleotide Excision Repair (NER) and Fanconi Anaemia (FA) help to repair the crosslinks where the latter one depends on the position of cell cycle. The repair pathways Homologous Recombination (HR) and NHEJ are involved in the DSBs repair.

ATM (ataxiatelangiectadia mutated kinase) phosphorylates the proteins required for HR, Fanconi Anaemia (FA) and NER and facilitates translesion synthesis. During HR and translesion synthesis, single stranded 
DNA (ssDNA) is formed which gets protected by RPA complex (RPA14, RPA70, RPA32) and BRCA1 and BRCA2. ${ }^{35}$ ATM triggers p53 dependent checkpoint kinase 2 arm (cHK2)/Cdc25A and MDM2/p53 arm whose activation leads to the inhibition of $\mathrm{Cdc} 25$ phosphorylation. It causes cyclin E dependent G1 cell cycle arrest. Activated ATM also leads to the formation of RAD51 nucleofilaments and the formation of RAD51/ gamma-H2AX foci. ${ }^{46}$

ATR (ATM -RAD3 related kinase) regulates the rate limiting step for NER and phosphorylation of checkpoints, thereby contributing to the cell cycle arrest. ${ }^{35}$ ATR is recruited to RPA-ssDNA complex and it interacts with the complex's canonical partners. ATR on activation, phosphorylates the various substrates involved in the downstream activities like check point kinase1, BRCA proteins. The steady state of these kinases is regulated by p300 (an acetyl transferase), which is a chief activator of DNA damage response. ${ }^{35}$ As mentioned above, if the damage can't be repaired, the cell undergoes apoptosis and ATM, ATR phosphorylates p53 for mediating the same. ${ }^{35,46}$

E6 has been found to act as cofactor that augment the genome destabilization by abrogating the DNA damage response which usually gets initiated in response to the UV radiation and by dysregulating the cell cycle (Figure 6). E6 binds with the p300 decreasing the steady state of the kinases and dysregulating all the processes. Due to the E6 oncoprotein, the repair of both cyclobutane pyrimidine dimer and the double strand breaks get delayed. Due to the degradation of p300 by E6, delayed foci resolution of gamma H2AX was observed. E6 attenuates the checkpoints' signalling, leading to the unscheduled cell cycle transition from G1 to S phase, letting the DNA damage unattended. ${ }^{35}$

\section{E7 contributing to the carcinogenesis}

E7 is a nuclear oncoprotein that has approximately 100 residues of amino acids. ${ }^{47}$ It has a $\mathrm{C}$-terminal zinc binding domain, the structure-based activity of which is very important for the E7 oncogene regulation. E7 has a $\mathrm{N}$-terminus that has similar portions to that of CR1 and the CR2, found in the Adenovirus E1AV as well as the sequences in the SV40 $\mathrm{T}$ antigen. The CR2 part of the E7 has LXCXE binding motif and binds to the protein like retinoblastoma tumor suppressor and by the ubiquitin ligase, targets it for degradation as shown in Figure 2. It does that by interconnecting with the cullin 2 ubiquitin ligase complex. Apart from that, the $\mathrm{N}$-terminal of the (E7) E7 protein, has Casein kinase II (CKII), which undergoes phosphorylation to drive the cell progression to S-phase. E7, by its LXCXE motif, even binds to the proteins p107 and p130 which are the pocket proteins with high affinity and downregulates them, further inactivating $\mathrm{pRb}$. E7 inhibits the negative control of cell transition from the G0 to the G1 phase. UBR4/p600 and p300/CBP-associated factor (P/CAF) have also been found to interact with the E7, driving the cell progression. E7 has also been involved in the dysregulation of the cell cycle by upregulating p16 and p21 stabilization. ${ }^{19}$ E7 has been known to induce DNA damage, chromosomal segregation defects and centrosome abnormalities. These lead to chromosomal instability. E7 has been seen to uncouple the cell cycle arrest and the differentiation by the inactivation of pRb. RB1, retinoblastoma susceptibility gene is a tumor suppressor gene. $\mathrm{pRb}$ is its gene product which is a negative controller of the cell cycle. pRb is a chromatin associated protein which, by the regulation of E2F transcription factors, limits the transcription of the genes involved in cell cycle. $\mathrm{pRb}$ also interconnects with the chromatin regulators to control the cell cycle. These interactions allow the $\mathrm{pRb}$ to recruit the complexes that repress the transcription process. By the suppression of E2F, $\mathrm{pRb}$ limits the expression of the proteins, involved in the proliferation of cell. The active $\mathrm{pRb}$ is initially in a partial state of latency. During the G1 phase, the arrest of cell cycle mediated by the checkpoints. When there occurs a hyperphosphorylation of the $\mathrm{pRb}$ in the G1 to $S$ phase's transition, it relieves the inhibitory activity of $\mathrm{pRb}$ on E2F allowing the cell progression to occur. The released E2F then leads to the Drp1 activation that further causes mitochondrial fission. ${ }^{48}$ Apart from repressing the E2F-regulated genes, $\mathrm{pRb}$ has also been involved in the chromosomal domains' organization especially in response to apoptosis and differentiation signals. The $\mathrm{pRb}$ gene not just targets the E2F, but it also has effects independent effects. pRb on interaction with Skp2 regulates the stability of $\mathrm{p} 27$. The cell cycle exits, regulated by the $\mathrm{pRb}$ is correlated in a better way with its effect on p27 rather than the changes in the protein expression of $\mathrm{E} 2 \mathrm{~F}$. The retinoblastoma protein is a component of one regulatory INK4A/Cyclin D1/pRb/E2F pathway that gets dysregulated during cancer. Hyperphosphorylated $\mathrm{pRb}$ interacts with the mTORC2 complex and attenuate the Akt activation. Hence, mutations in the $\mathrm{pRb}$ can alter the differentiation programmes. Studies have shown that viral oncoproteins directly target the $\mathrm{pRb}$ hence promoting the viral replication. ${ }^{3} \mathrm{pRb}$ loss has led to the defects that occur in the cell cycle during cell exit, entry into the division phase, compromising with the G1/S arrest and therefore reducing senescence. Myc has also been found to play a major role with the pathways involving the altered $\mathrm{pRb}$. $\mathrm{pRb}$ loss increases the inci- 


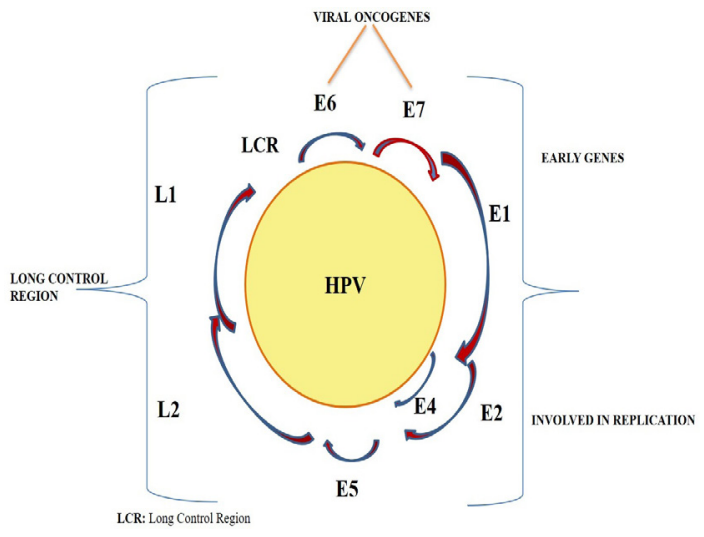

Figure 1: The early proteins are responsible for the regulation of viral replication process are consists of E1, E2, E4, E5, E6 and E7. The LCR has several sites for the binding of proteins to activate viral expression with L1 and L2 as its major and minor capsid proteins respectively. LCR: Long control region.
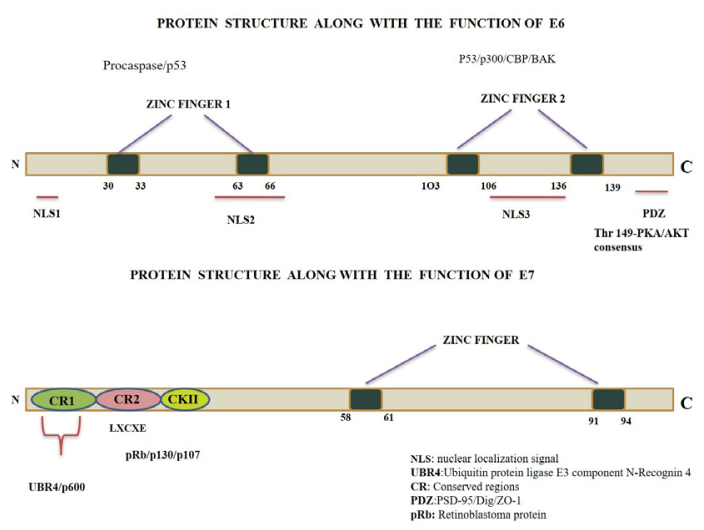

Figure 2: The E7 gene by its LXCXE motif binds to the p107 and p130, inactivating the pRb. Casein kinase II is associated with phosphorylation that causes $\mathrm{S}$ phase transition. The UBR4/p600 too drives the cell progression. All the NLSs in the E6 oncogene impair the apoptotic proteins. UBR4: Ubiquitin protein ligase E3 Component N-Recognin 4, NLSs: Nuclear localization signals, pRb: Retinoblastoma protein.

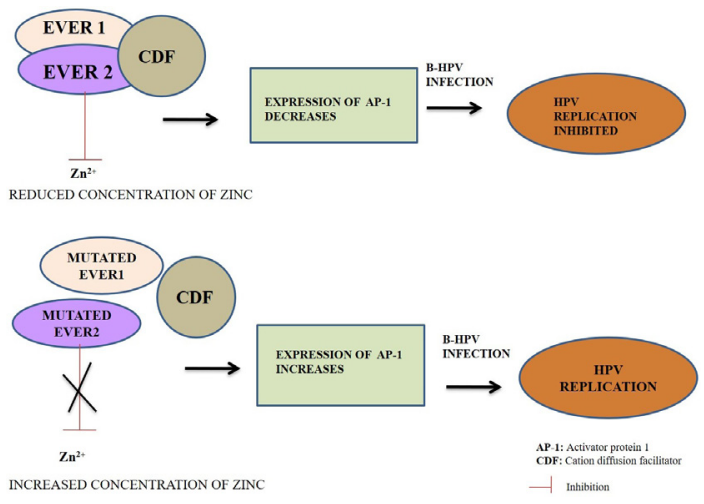

Figure 3: The EVER/Zn-T or the EVER/CDF complex regulate the zinc homeostasis regulating the expression of growth factors by zinc translocation. The mutated EVER genes or the virus infected EVER genes are unable to perform their respective function thus stimulating the expression of AP-1 by JNK activation that causes the infected cells to replicate. AP-1: Activator protein, Zn-T: Zinc Transporter, CDF: Cation diffusion facilitator, JNK: c-Jun N-terminal kinase.

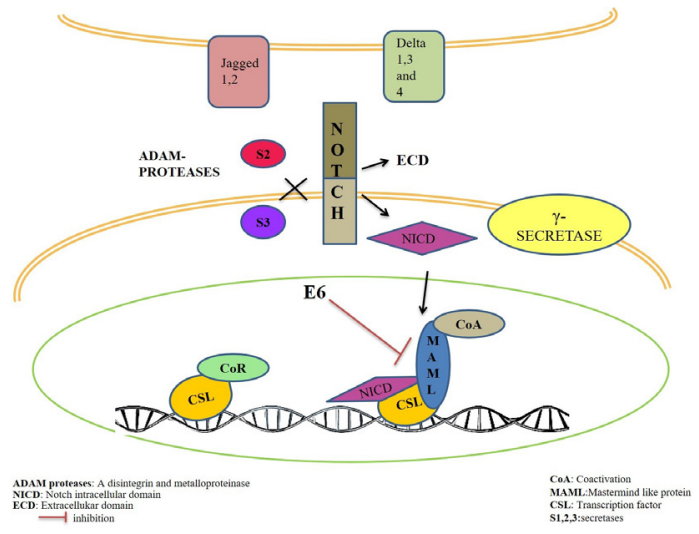

Figure 4: E6 protein binds to the LXXLL motif of MAML and prevents the complex RBPJ/ICN/MAML to function for the differentiation of cells. MAML: Mastermind like protein RBPJ: Recombining binding protein suppressor of hairless, ICN: Intracellular domain.

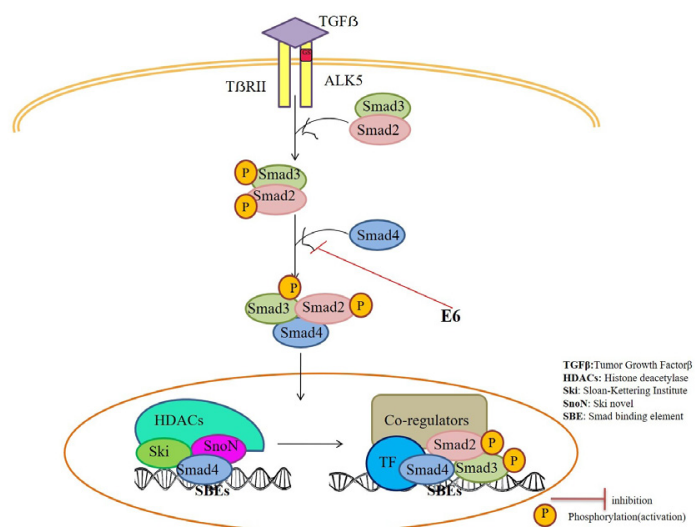

Figure 5: E6 protein doesn't allow the interaction of Smad 4 to occur with Smad2/3 thereby preventing the transcriptional activation for the TGF- $\beta$ involved in the differentiation of cells. TGF- $\beta$ : Transforming growth factor/Tumor growth factor $\beta$.

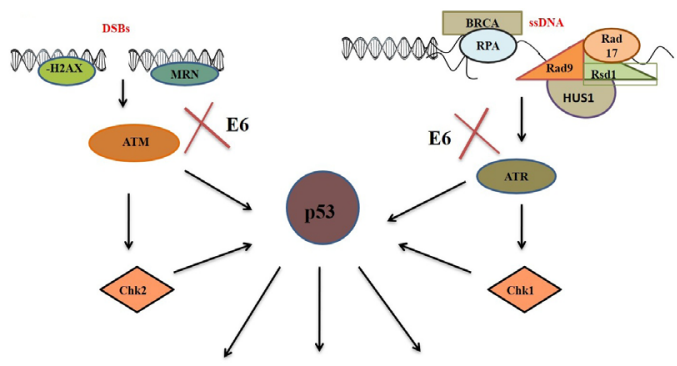

APOPTOSIS, CELL CYCLE ARREST, SENESCENCE, DNA REPAIR

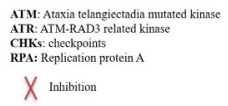

Figure 6: Due to the E6 viral protein, the activity of ATR, ATM, BRCAs, RPA proteins get impaired, that target the $\mathrm{p53}$. The checkpoints fail to get activated to carry out the cell cycle arrest of cells with damaged DNA. ATM: ataxia telangiectadia mutated kinase, ATR: ATM-RAD3 related kinase, RPA: Replication protein A, BRCA: Breast cancer genes. 


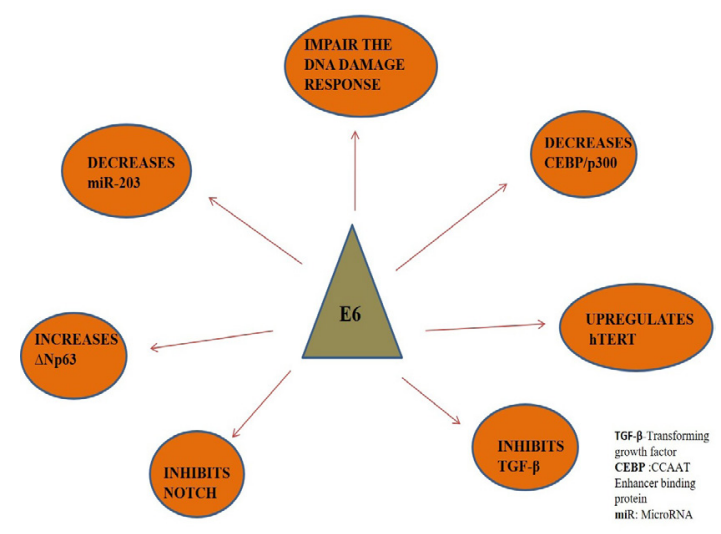

Figure 7: E6 targets all the proteins that activate the apoptotic proteins. It increases the pool of proteins that antagonize the proteins involved in cell death.

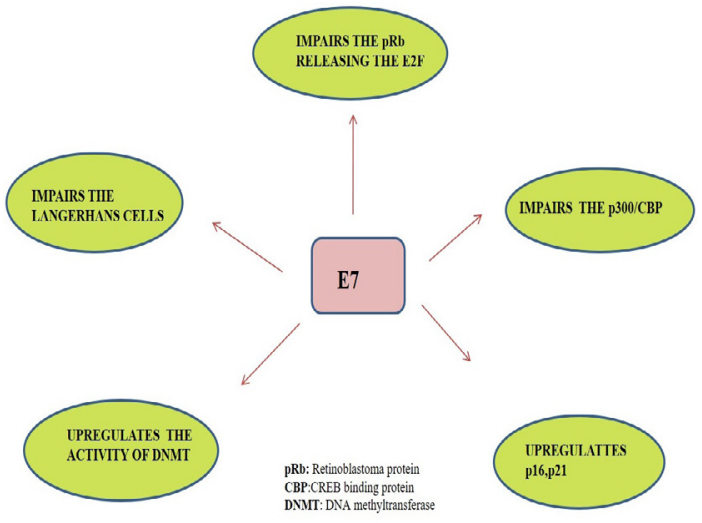

Figure 8: The E7 oncogene primarily target the pRb and increases cell proliferation. It even decreases the expression of APCs impairing the pathways involved in apoptosis. APCs: Antigen presenting cells (Langerhans cells), pRb: Retinoblastoma protein.

dences of lagging chromosomes and reduces the fidelity of chromosome segregation. These lead to the mutations in $\mathrm{p} 53$. pRb loss is linked to the mitotic phenotypes such as altered expression of the mitotic protein, less Condensin 11 protein CapD3, decreased cohesion of chromosomes and an alteration in the accumulation of cohesion complexes at the pericentromeric chromatin. The E7 oncogene causes the inactivation of $\mathrm{pRb}$ when the cell is in its proliferative state. ${ }^{3}$ E7 interacts with $\mathrm{pRb}$ causing its degradation. It causes the release of E2F transcriptional factor that is responsible for triggering the expression of cyclins and the other S-phase regulators. E7 links itself with the other proteins that are involved in the division of cells. It involves the histone acetylases, components of the AP-1 transcription complex and the cyclin dependent kinase inhibitors $\mathrm{p} 21$ and $\mathrm{p} 27 .{ }^{19}$ The $\mathrm{pRb}$ protein is located in the outer membrane of the mitochondria. It interacts physically with the Bax and favours apoptosis. This suggests that $\mathrm{pRb}$ loss can also affect mitochondrial functions. The $\mathrm{pRb}$ is

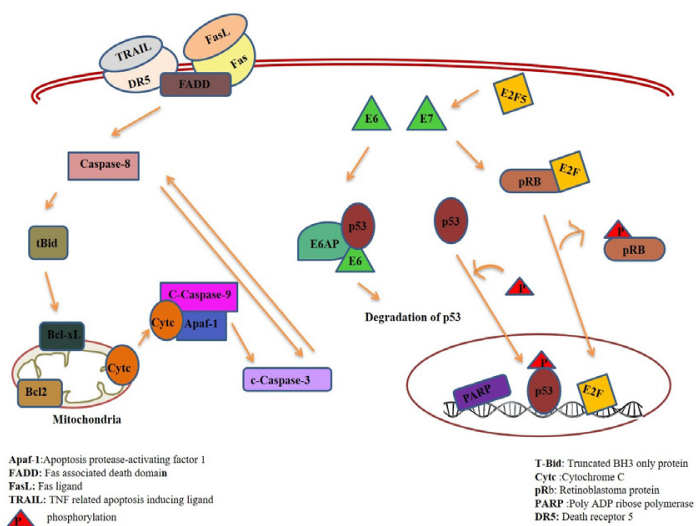

Figure 9: The E7 displaces the E2F from the pRb-E2F complex and this free E2F then promotes the cell cycle progression. The E6 targets the p53 impairing a number of apoptotic pathways. The E6 targets the BAK expression and promote the cell survival. BAK: Bcl-2 killer, pRb: Retinoblastoma protein.

one of the most salient targets of the $\mathrm{Cdk} 4 / 6$ kinases. The $\mathrm{pRb}$ gets activated in the G1 phase, by the inhibitors of $\mathrm{Cdk} 4 / 6$. These inhibitors on combining with other inhibitors like HER2, PI3K/mTOR and B-RAF, MEK and IGFIR/IR and produce a synergic effect. ${ }^{3} \mathrm{It}$ was found that despite high level of p21, E7 was able to abrogate the DNA damage response. It prevented p21 from inhibiting the $\mathrm{Cdk} 2 /$ cyclin $\mathrm{E}$ activity and the PCNA-dependent DNA replication. ${ }^{48}$ E7 forms a complex with AP-1 transcriptional factors Jun B, c Fos and c-Jun. E7 zinc fingers are involved in the regulatory pathways of AP-1 transcription factors and not the pRb binding site. Using the c-Jun deletion mutants, the E7 can bind maps between the amino acids 224 and 286 of c-Jun.E7 then transactivates the transcription which is induced by the c-Jun with the help of a Jun responsive promoter. $^{49}$

\section{E7: A stimulator of DNA methyltransferase}

In the tumor cells, an increased activity of DNA methyl transferase1 (DNMT1) was seen with DNA hypomethylation in the repetitive regions and hypermethylation in the $\mathrm{cpG}$ islands of the tumor suppressor gene promoter. E7 has been found to bind to the DNMT1, stimulating its activity. Activation of DNMT1 occurs by the pRb/ E2F pathway. The most frequent methylated genes are cell adhesion molecule 1(CADM1), cadherin, cyclin A1 (CCNA1), Erythrocyte membrane protein band 4,1 like 3 (EPB41L3), death associated protein kinase 1 (DAPK1), myelin and lymphocyte (MAL), telomerase reverse transcriptase (hTERT). Due to the methylation, the Ras associated domain family member 1 (RASSF1) gets downregulated which is a key regulator of apoptosis. Due to the methylation, CADM1 gets silenced in its promoter region, hence causing metastasis. The 
CDH1 gets downregulated due to methylation due to the presence of the viral oncogene. Another protein is the PRDM14 that gets hypermethylated. The DAPK, a pro-apoptotic serine/threonine kinase gets downregulated that plays a major role in metastasis and tumor progression. Further, methylation of the decoy receptors DcR1, DcR2 was reported that lead to the apoptosis inhibition. ${ }^{19}$

\section{E7 dysregulating the histone modification}

HPV E7 has also been shown to modify the competence of transcription of the infected cells by modulating the activities of histone-modifying and chromatin re-modelling enzymes. Lysine residues of the histone 3 and histone 4 (H3 and H4) get acetylated by the histone deacetylase (HDACs) that result in the transcriptionally active chromatin whereas the deletion of these marks by the histone deacetylases, lead to the transcriptionally suppressed chromatin. E7 associates with the enzymes that modulate the histone deacetylase. E7 can even bind to the p300/CBP and lead to its acetylation. The acetylation hence decreases the pool of p300/CBP. E7 also binds to the p300/CBP0 Association Factor (pCAF) association factor hence decreasing its ability to acetylate the histones. Class 1 of the histone deacetylase functions as the co-repressor of transcription by bringing out the reversal of acetyl modifications on the lysine residue of histone. This causes chromatin remodelling. The association of E7 with the HDACs1/2 occur by the Mi2 $\beta$ which is a member of the nucleosome remodelling and the histone deacetylation complex $(\mathrm{NuRD})$ and not in a $\mathrm{pRb}$ dependent manner. The NuRD, repositions the ATP-dependent nucleosomes and deacetylates the histones for the remodelling of the chromatin structure. The E7 was found to interlink with the interferon response factor 1 (IRF1) and cause the recruitment of HDACs to suppress the IRF1's transcriptional activity. E7 was found to block the activity of HDACs on Hypoxia Inducible Factor-1 (HIF-1) which can lead to the activation of a wide range of angiogenic factors. DNA methylation and modifications in histone acetylation were also found to repress the retinoic acid repressor $\beta 2$ (RAR- $\beta 2$ ) and proliferation repressor protein osteoprotegerin (OPG). The E7 also share its role in the histone methylation. Histone methylation is controlled by two major enzymes: histone lysine demethylase (KDMs) and histone methyl transferase (HMTs). KDMs regulate the level of methylation and contribute to the gene expression regulation by either suppression or activation. The HMTs methylates the histone on different lysine residues. However, the KDMs expression's downregulation has been seen to exacerbate the carcinogenesis process. E7 can induce transcriptional induction of the KDM6A and KDMB histone 3 lysine 27 (H3K27)-specific demethylase. ${ }^{19}$

\section{E7 affecting the E-cadherin}

E Cadherin, a cell adhesion protein, dependent on calcium, is used by the Langerhans cells that move via the stratified epithelium as a response of immune system. E7 can reduce the E Cadherin level by depending on cellular DNA methyl transferase 1 (Dnmt1) by increasing its expression and not depending on AP- $2 \alpha$ or the $\mathrm{pRb}$ degradation. The HPV can create a zone within the infected keratinocytes that can be resistant to the antigen presenting cells- the Langerhans cells. ${ }^{50}$ Langerhans cells are the skin homing dendritic cell which are immature. They are expressed in the uppermost layers of the differentiated epithelial cells. CCR6 is the precursor of Langerhans cells that directs them along a gradient. ${ }^{51}$ They entrap the antigens and display to the immune system. To recognize the antigens, they can cross the prime $\mathrm{T}$ cells. Whenever there is an encounter of antigen, the process of endocytosis occurs to internalize them. Their foreign peptides get processed and then get displayed on the class 1 or class 2 major histocompatibility complex (MHC). This allows the T-helper and the cytotoxic cells to display the antigens. Further, the secretion of IL-12 cytokine stimulates the natural killer cells (NK) and destroy the foreign particles (antigens). ${ }^{52}$ Migration of these antigen presenting cells gets influenced by a lot of factors like granulocyte macrophage colony stimulating factor, interleukins, Tumor Necrosis Factor whose levels get altered by this oncoprotein. To be present in the keratinocyte membrane, the antigen presenting cells need the assistance of $\mathrm{E}$ Cadherin. The usual activity of the E7 is to target the pRb. Hence, earlier it was thought that E7 targets $\mathrm{pRb}$ and AP-2 $\alpha$ to degrade. However, studies showed that the level of $\mathrm{E}$ Cadherin was found out to be unchanged when the $\mathrm{pRb}$ was directed for degradation. ${ }^{50} \mathrm{C} / \mathrm{EBP} \beta$ is a regulator of CCL2. $\mathrm{E} 7$ has found to be in proximity with it and impair its binding with the CCL20 promoter region. CCL20, a cc chemokine ligand 20, also called as the macrophage inflammatory protein- $3 \alpha$ works to recruits the Langerhans cells. Hence, E7 binding with the C/EBP $\beta$ decreases the ability of the CCL20 to recruit the Langerhans for the antigen presentation. ${ }^{51} \mathrm{NF}-\mathrm{kB}$ has also been found to associate with CCL20 as the NF-kB site is situated between the $\alpha \mathrm{C} / \mathrm{EBP}$ - binding site that is important for Poly (1:c)'tumor necrosis factor (TNF), IL- $1 \alpha$ and IL-1 $\beta$ that induces the CCL20 gene expression. ${ }^{51}$ 


\section{CONCLUSION}

Epidermodysplasia Verruciformis has been a tropical disease to link viral infection with carcinogenesis. The EVER genes, EVER1 and EVER2 when get mutated, are not able to cooperate with the $\mathrm{Zn}-\mathrm{T}$ complex for maintaining the zinc homeostasis. The attack of Human Papillomavirus even in the normal keratinocytes has been involved in the function of transforming the activities of EVER genes in zinc regulation. This increase in the zinc ion concentration drives to provide an expedient environment for the activation of cell proliferation pathways such as JNK pathway and inhibiting the apoptotic ones such as TRAIL induced apoptosis. The excess zinc is able to bind to the zinc fingers of E6 and E7 viral oncogenes that act as secondary messenger for the proliferation of infected keratinocytes. E6 and E7 have been majorly involved in the dysregulation of a wide range of signalling pathways (Figure 9). E6 has been found to affect many pathways by destabilizing the $\mathrm{p} 53$ by histone modification. It acts as a cofactor of UV induced cancer where it prevents the activation of ATM and ATR and checkpoints' activation that therefore prevents the DNA damage repair. E6 dysregulates the interaction between p300, C/EBP and miR-203 decreasing the pool of antiapoptotic proteins. Most importantly, E6 affects the Notch and the TGF- $\beta$ pathways by preventing the interaction of ICN-MAML-RBPJ and Smad2/3 with Smad respectively. On the contrary the oncogene E7 has primarily been responsible for causing the displacement of $\mathrm{pRb}$ from E2F and inactivating $\mathrm{pRb}$. E7 can upregulate p16 and p21 and delay the senescence of infected keratinocytes. $\mathrm{E} 7$ is capable of dysregulating the $\mathrm{CB} / \mathrm{p} 300$ by altering their acetylation process. $\mathrm{E} 7$, by binding to the C/EBP- $\beta$, impairs the recruitment of Langerhans cells and hence impairs the immune response to virus. E7 also upregulates the activity of DNA methyl transferase and inhibits the activities of various proteins involved in apoptosis (Figure 8) The invasion by HPV in the keratinocytes in a way upregulates the signal transduction involved in the proliferation, preventing the apoptosis. Hence, our target should be the inhibition of E6's and E7's expression that causes the malignancy to occur.

\section{ACKNOWLEDGEMENT}

The authors highly appreciate the immense support provided by the management of Birla Institute of Technology, Mesra (Ranchi, India) and acknowledge the facilities provided.

\section{CONFLICT OF INTEREST}

The authors declare that there are no conflicts of interest.

\section{ABBREVIATIONS}

HPV: Human Papilloma Virus; EV: Epidermodysplasia Verruciformis; NCR: Non-coding region; LCR: Long coding region; NK: Natural killer cells; TNF: Tumor Necrosis Factor; HDACs: Histone deacetylase; HMTs: Histone methyl transferase; HIF-1: Hypoxia Inducible Factor-1.

\section{REFERENCES}

1. Lazarczyk M, Cassonet P, Pons C, Jacob Y, Favre M. The EVER proteins as a natural barrier against Papillomaviruses: A new insight into the pathogenesis of human papillomavirus infections. Microbiology and Molecular Biology Reviews. 2009;73(2):348-70.

2. Vinzón SE, Braspenning-Wesch I, Müller M, Geissler EK, Nindl I, et al. Protective vaccination against papillomavirus-induced skin tumors under immunocompetent and immunosuppressive conditions: A preclinical study using a natural outbred animal model. PLoS Pathogens. 2014;10(2): e1003924.

3. Dyson NJ. RB1: A prototype tumor suppressor and an enigma. Genes and Development. 2016;30(13):1492-502.

4. Saraiya M, Unger ER, Thompson TD, Lynch CF, Hernandez BY, et al. US assessment of HPV types in cancers: Implications for current and 9-valent HPV vaccines. JNCl: Journal of the National Cancer Institute. 2015;107(6):112.

5. Kalińska-Bienias A, Kowalewski C, Majewski S. The EVER genes-the genetic etiology of carcinogenesis in epidermodysplasia verruciformis and a possible role in non-epidermodysplasia verruciformis patients. Advances in Dermatology and Allergology/Postẹpy Dermatologii I Alergologii. 2016;33(2):75.

6. Imahorn E, Yuksei Z, Spoerri I, Gürel G, Imhof C, et al. Novel TMC 8 splice site mutation in epidermodysplasia verruciformis and review of HPV infections in patients with the disease. Journal of the European Academy of Dermatology and Venereology. 2017;31(10):1722-6.

7. Lutzner MA. Epidermodysplasia verruciformis. An autosomal recessive disease characterized by viral warts and skin cancer. A model for viral oncogenesis. Bulletin Du Cancer. 1978;65(2):169-82.

8. Aksal PGML, et al. Tree man syndrome: A review. International Journal of Allied Medical Sciences and Clinical Research. 2016;4:171-6.

9. Leiding JW, Holland SM. Warts and all: Human papillomavirus in primary immunodeficiencies. Journal of Allergy and Clinical Immunology. 2012;130(5):1030-48.

10. lannacone MR, Gheit T, Pfister H, Giuliano AR, Messina JL, et al. Casecontrol study of genus-beta human papillomaviruses in plucked eyebrow hairs and cutaneous squamous cell carcinoma. International Journal of Cancer. 2014;134(9):2231-44.

11. Vandeven N, Ngheim P. Pathogen-driven cancers and emerging immune therapeutic strategies. Cancer Immunology Research. 2014;2(1):9-14.

12. Aldabagh B, Angeles JGC, Cardones AR, Arron ST. Cutaneous squamous cell carcinoma and human papillomavirus: Is there an association?. Dermatologic Surgery. 2013;39(1pt1):1-23.

13. Viarisio D, Muller-Decker K, Accardi R, Robitaille A, Dürst M, et al. Beta HPV38 oncoproteins act with a hit-and-run mechanism in ultraviolet radiationinduced skin carcinogenesis in mice. PLoS Pathogen. 2018;14(1):e1006783.

14. Chai RC, Lambie D, Verma M, Punyadeera C. Current trends in the etiology and diagnosis of HPV-related head and neck cancers. Cancer Medicine. 2015;4(4):596-607.

15. Harwood CA, McGregor JM, Proby CM, Breuer J. Human papillomavirus and the development of non-melanoma skin cancer. Journal of Clinical Pathology. 1999;52(4):249-53.

16. Fuchs PG, Iftner T, Weninger J, Pfister HE. Epidermodysplasia verruciformisassociated human papillomavirus 8: Genomic sequence and comparative analysis. Journal of Virology.1986;58(2):626-34.

17. Litwin TR, Clarke MA, Dean M, Wentzensen N. Somatic host cell alterations in HPV carcinogenesis. Viruse. 2017;9(8):206. 
18. Gavioli CF, Festo NC, Tyring SK, Silva LL, Oliveira WR. High-risk mucosal HPV types associated with squamous cell carcinoma on the nose tip in an immunocompetent young man. Anais Brasileiros De Dermatologia. 2018;93(5):716-8.

19. Gupta SM, Mania-Pramanik J. Molecular mechanisms in progression of HPV-associated cervical carcinogenesis. Journal of Biomedical Science. 2019;26(1):28.

20. White EA, Walther J, Javanbakht H, Howley PM. Genus beta human papillomavirus E6 proteins vary in their effects on the transactivation of p53 target genes. Journal of Virology. 2014;88(15):8201-12.

21. Emsen IM, Kabalar ME. Epidermodysplasia verruciformis: An early and unusual presentation. Canadian Journal of Plastic Surgery. 2010;18(1):21-4.

22. Kaushal A, Silver S, Keynan Y, Severini A, Hamza S. Epidermodysplasia verruciformis in an HIV-infected man: A case report and review of the literature. Topics in Antiviral Medicine. 2012; 20(5):173.

23. Orth G. Genetics of epidermodysplasia verruciformis: Insights into host defense against papillomaviruses. In Seminars in Immunology. Academic Press. 2006;18(6):362-74.

24. Keresztes $G$, Mutai $H$, Heller S. TMC and EVER genes belong to a larger novel family, the TMC gene family encoding transmembrane proteins. BMC Genomics. 2003;4(1):24.

25. Kurima K, Yang Y, Sorber K, Griffith AJ. Characterization of the Transmembrane Channel like (TMC) gene family: Functional clues from hearing loss and epidermodysplasia verruciformis. Genomics. 2003;82(3):300-8.

26. Choi S, Hu Y, Corkins M, Palmer AE, Bird AJ. Zinc transporters belonging to the Cation Diffusion Facilitator (CDF) family have complementary roles in transporting zinc out of the cytosol. PLoS Genetics. 2018;14(3):e1007262.

27. DeJong SJ, Imahorn E, Itin P, Hum D, Gunasekharan V, et al. Epidermodysplasia verruciformis: Inborn errors of immunity to human betapapillomaviruses. Frontiers in Microbiology. 2018;9:1-9.

28. Robertson $\mathrm{H}$, Hayes $\mathrm{J}$, Sutherland $\mathrm{C}$. A partnership with the proteasome: The destructive nature of GSK3. Biochemical Pharmacology. 2018;147:77-92.

29. Gaud G, Guillemot D, Jacob Y, Favre M, Vuillier F. EVER2 protein binds TRADD to promote TNF- $\alpha$-induced apoptosis. Cell Death and Disease. 2013;4(2):e499.

30. Leisner TM, Freeman T, Black JL, Parise LV. CIB1: A small protein with big ambitions. The FASEB Journal. 2016;30(8):2640-50.

31. Notarangelo LD. HPV: CIB1 is for EVER and EVER. Journal of Experimental Medicine. 2018;2229-31.

32. DeJong SJ, Crequer A, Matos I, Hum D, Gunasekharan V, et al. The human CIB1-EVER1-EVER2 complex governs keratinocyte-intrinsic immunity to $\beta$-papillomaviruses. Journal of Experimental Medicine. 2018;215(9):2289310.

33. Boon SS, Tomaic V, Thomas M, Roberts S, Banks L. Cancer-causing human papillomavirus E6 proteins display major differences in the phosphoregulation of their PDZ interactions. Journal of Virology. 2015;89(3):1579-86.

34. Stubenrauch F, Leigh IM, Pfister H. E2 represses the late gene promoter of human papillomavirus type 8 at high concentrations by interfering with cellular factors. Journal of Virology. 1996;70(1):119-26.

35. Wendel SO, Wallace N. Loss of genome fidelity: Beta HPVs and the DNA damage response. Frontiers in Microbiology. 2017;8:1-19.
36. Nunes EM, Nunes VT, Sichero L. Epidemiology and biology of cutaneous human papillomavirus. Clinics. 2018;73:1-9.

37. Meyers JM, Uberoi A, Grace M, Lambert PF, Munger K. Cutaneous HPV8 and MmuPV1 E6 proteins target the NOTCH and TGF- $\beta$ tumor suppressors to inhibit differentiation and sustain keratinocyte proliferation. PLoS Pathogens. 2017;13(1):e1006171.

38. Marthaler AM, Podgorska M, Feld P, Fingerle A, Knerr-Rupp K, et al. Identification of C/EBPa as a novel target of the HPV8 E6 proteinregulating miR-203 in human keratinocytes. PLoS Pathogens. 2017;13(6):e1006406.

39. Daniel R, Wu Q, Williams V, Clark G, Guruli G, et al. A panel of MicroRNAs as diagnostic biomarkers for the identification of prostate cancer. International Journal of Molecular Sciences. 2017;18(6):1281.

40. Tucci P, Agostini M, Grespi F, Markert EK, Terrinoni A, et al. Loss of p63 and its microRNA-205 target results in enhanced cell migration and metastasis in prostate cancer. Proceedings of the National Academy of Sciences. 2012;109(38):15312-7.

41. Nekulova M, Holcakova J, Coates P, Vojtesek B. The role of p63 in cancer, stem cells and cancer stem cells. Cellular and Molecular Biology Letters. 2011;16(2):296.

42. Müller-Schiffmann A, Beckmann J, Steger G. The E6 protein of the cutaneous human papillomavirus type 8 can stimulate the viral early and late promoters by distinct mechanisms. Journal of Virology. 2006;80(17):8718-28.

43. Meyers JM, Grace M, Uberoi A, Lambert PF, Munger K. Inhibition of TGF- $\beta$ and NOTCH signaling by cutaneous papillomaviruses. Frontiers in Microbiology. 2018;9:1-11.

44. Vanorny DA, Mayo K. The role of Notch signaling in the mammalian ovary. Reproduction. 2017;153(6):187-204.

45. Mendoza JA, Jacob Y, Cassonnet P, Favre M. Human papillomavirus type 5 E6 oncoprotein represses the transforming growth factor $\beta$ signaling pathway by binding to SMAD3. Journal of Virology. 2006;80(24):12420-4.

46. Hong SY. DNA damage response is hijacked by human papillomaviruses to complete their life cycle. Journal of Zhejiang University-SCIENCE B. 2017;18(3):215-32.

47. Roman A, Munger K. The papillomavirus E7 proteins. Virology. 2013;445(12):138-68.

48. Thomas RJ, Oleinik N, Selvam SP, Vaena SG, Dany M, et al. HPV/E7 induces chemotherapy-mediated tumor suppression by ceramide-dependent mitophagy. EMBO Molecular Medicine. 2017;9(8):1030-51.

49. Antinore MJ, Birrer M, Patel D, Nader L, McCance DJ. The human papillomavirus type $16 \mathrm{E} 7$ gene product interacts with and transactivates the AP1 family of transcription factors. The EMBO Journal. 1996;15(8):1950-60.

50. Laurson J, Khan S, Chung R, Cross K, Raj K. Epigenetic repression of E-cadherin by human papillomavirus 16 E7 protein. Carcinogenesis. 2010;31(5):918-26.

51. Sperling $T$, Oldak $M$, Walch-Rückheim B, Wickenhauser $C$, Doorbar J, et al. Human papillomavirus type 8 interferes with a novel C/EBP $\beta$-mediated mechanism of keratinocyte CCL20 chemokine expression and Langerhans cell migration. PLoS Pathogens. 2012;8(7):e1002833.

52. Mutyambizi K, Berger C, Edelson RL. The balance between immunity and tolerance: The role of Langerhans cells. Cellular and Molecular Life Sciences. 2009;66(5):831-40.

\section{SUMMARY}

Increase in the zinc ion concentration because of the mutations found in EVER genes of keratinocytes, dysregulate the zinc homeostasis and increase the downstream action of JNK and decreases the TRAIL induced apoptosis. The excess zinc is able to bind to the zinc finger motifs of E6 and E7 oncoproteins of HPV that increases the cell proliferative pathways. E6 oncoproteins, even independent of EVER genes are involved in dysregulation od DDR on exposure to UV, NOTCH, TGF signalling pathways and even affect other genes involved in cell survival and death of keratinocytes. E7 in the same way promotes the antiapoptotic genes to get transcribed and thereby foster skin carcinogenesis. 


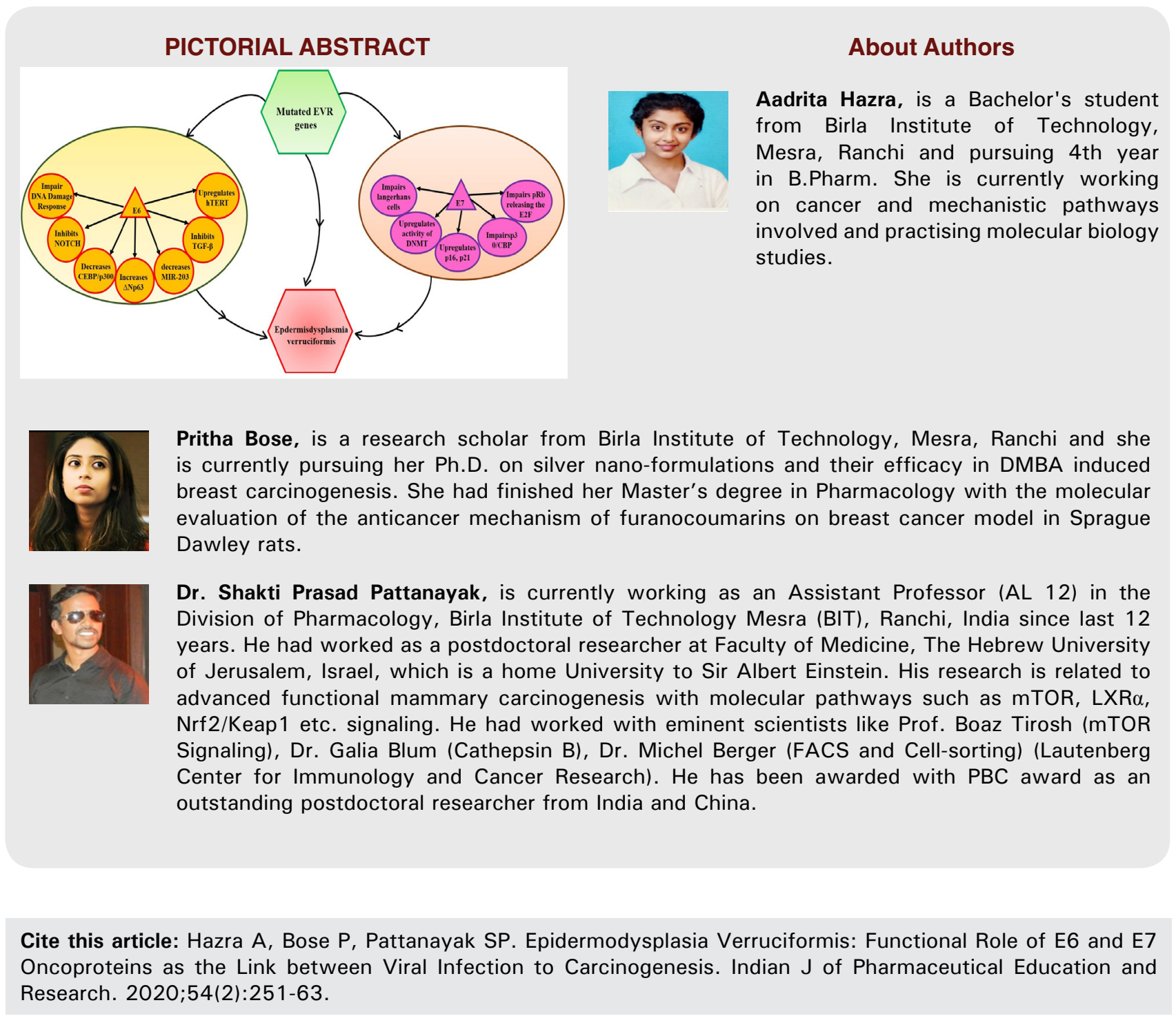

\section{PICTORIAL ABSTRACT} is currently pursuing her Ph.D. on silver nano-formulations and their efficacy in DMBA induced breast carcinogenesis. She had finished her Master's degree in Pharmacology with the molecular evaluation of the anticancer mechanism of furanocoumarins on breast cancer model in Sprague Dawley rats.

Dr. Shakti Prasad Pattanayak, is currently working as an Assistant Professor (AL 12) in the Division of Pharmacology, Birla Institute of Technology Mesra (BIT), Ranchi, India since last 12 years. He had worked as a postdoctoral researcher at Faculty of Medicine, The Hebrew University of Jerusalem, Israel, which is a home University to Sir Albert Einstein. His research is related to advanced functional mammary carcinogenesis with molecular pathways such as mTOR, LXR $\alpha$, Nrf2/Keap1 etc. signaling. He had worked with eminent scientists like Prof. Boaz Tirosh (mTOR Signaling), Dr. Galia Blum (Cathepsin B), Dr. Michel Berger (FACS and Cell-sorting) (Lautenberg Center for Immunology and Cancer Research). He has been awarded with PBC award as an outstanding postdoctoral researcher from India and China. Research. 2020;54(2):251-63. 